\title{
Monte Carlo Methods for the Nuclear Shell Model
}

\author{
C. W. Johnson, S. E. Koonin, G. H. Lang, and W. E. Ormand \\ W. K. Kellogg Radiation Laboratory, California Institute of Technology, Pasadena, California 91125
}

(Received 31 August 1992)

\begin{abstract}
We present novel Monte Carlo methods for treating the interacting shell model that allow exact calculations much larger than those heretofore possible. The two-body interaction is linearized by an auxiliary field; Monte Carlo evaluation of the resulting functional integral gives ground-state or thermal expectation values of few-body operators. The "sign problem" generic to quantum Monte Carlo calculations is absent in a number of cases. We discuss the favorable scaling of these methods with nucleon number and basis size and their suitability to parallel computation.
\end{abstract}

PACS numbers: $21.60 . \mathrm{Cs}, 02.70 .+\mathrm{d}, 21.60 . \mathrm{Ka}$

The shell model (valence fermions confined by a onebody potential and influencing each other through a residual two-body interaction) is a ubiquitous framework for the quantum many-body problem and is often the method of choice for describing nuclear structure [1]. For example, the low-lying spectra and one-body transition matrix elements of nuclei with $17 \leq A \leq 39$ are very well described by the exact diagonalization of an effective twobody Hamiltonian within the single-particle basis of the $1 d_{5 / 2}-1 d_{3 / 2}-2 s_{1 / 2}$ orbitals [2].

Unfortunately, the combinatorial growth of the dimension of the many-body basis with both the number of valence particles $\left(N_{V}\right)$ and the size of the single-particle basis $\left(N_{S}\right)$ precludes an exact treatment for most larger nuclei and forces what are often ad hoc truncations of the many-body basis. The $J^{\pi}=0^{+}, T=0$ states of ${ }^{28} \mathrm{Si}$ are obtained by constructing and diagonalizing an $839 \times 839$ Hamiltonian matrix in the $s d$-shell basis noted above. A similar calculation for ${ }^{60} \mathrm{Zn}$ in the middle of the next major shell (ten neutrons and ten protons in the $1 f_{7 / 2}-1 f_{5 / 2}-2 p_{3 / 2}-2 p_{1 / 2}$ orbitals) would increase the dimension to $5,053,574$ and is clearly beyond the reach of today's computers.

In this Letter, we discuss Monte Carlo methods for the exact treatment of a shell-model Hamiltonian, $H$. They are based on using the imaginary-time, many-body evolution operator, $\exp (-\beta H)$, either to define the canonical or grand canonical ensemble at a temperature $\beta^{-1}$ or, for large $\beta$, to filter a many-body trial state to the exact ground state; thus both thermodynamic and groundstate properties can be obtained. Relative to direct diagonalization, these methods scale much more gently with $N_{S}$ and/or $N_{V}$, and hence hold the promise of extending complete shell-model calculations to much larger systems.

Our methods broadly follow previous work on the Hubbard model [3] and coordinate-space fermion systems [4], but differ significantly in detail due to the peculiarities of the nuclear shell model. A general expression for $H$ is

$$
H=\sum_{\alpha} \epsilon_{\alpha} \mathcal{O}_{\alpha}+\frac{1}{2} \sum_{\alpha} V_{\alpha} \mathcal{O}_{\alpha}^{2}
$$

where the $\mathcal{O}_{\alpha}$ are a set of noncommuting one-body oper- ators, the $c$ numbers $\epsilon_{\alpha}$ are related to the single-particle energies, and the $c$ numbers $V_{\alpha}$ characterize the residual interaction. A minimal choice for the $\mathcal{O}_{\alpha}$ is the set of Hermitian and anti-Hermitian parts of the multipole density operators, $\rho_{\mathrm{KM}}=\left[a^{\dagger} \times \tilde{a}\right]_{\mathrm{KM}}$. In this case, the $V_{\alpha}$ are related by a Pandya transformation to the usual twobody matrix elements of the residual interaction. However, even for a fixed $H$, there is considerable freedom in writing Eq. (1). For example, arbitrary symmetrized two-body matrix elements of the residual interaction have no effect on the (antisymmetric) eigenstates and eigenvalues of $H$, but do change the $V_{\alpha}$. Additionally, the density operators could be supplemented by the suitably Hermitized pair operators $\Delta_{\mathrm{JM}}^{\dagger}=\left[a^{\dagger} \times a^{\dagger}\right]_{\mathrm{JM}}$ and their adjoints, which might be convenient given the strong pairing character of the residual interaction.

For a concrete illustration, we consider refining a trial determinant $|\Psi\rangle$ to the exact ground state; details of implementation and other applications will be given elsewhere [5]. For any observable operator $O$, we define

$$
\langle O\rangle=\frac{\left\langle\Psi\left|e^{-\beta H / 2} O e^{-\beta H / 2}\right| \Psi\right\rangle}{\left\langle\Psi\left|e^{-\beta H}\right| \Psi\right\rangle} .
$$

In the limit $\beta \rightarrow \infty,\langle O\rangle$ approaches the required groundstate expectation value (as long as $|\Psi\rangle$ is not orthogonal to the true ground state). We divide the "time" $\beta$ into an even number, $N_{t}$, of equal intervals of duration $\Delta \beta=$ $\beta / N_{t}$ and introduce a real auxiliary field $\sigma_{\alpha n}$ coupled to each operator $\mathcal{O}_{\alpha}$ at each time slice $n=1, \ldots, N_{t}$. Then, in the limit $\Delta \beta \rightarrow 0$, the Hubbard-Stratonovich transformation allows $\langle O\rangle$ to be written as

$$
\langle O\rangle=\frac{\int \mathcal{D}[\sigma] W(\sigma) \Phi(\sigma) O(\sigma)}{\int \mathcal{D}[\sigma] W(\sigma) \Phi(\sigma)} .
$$

Here, $\mathcal{D}[\sigma]=\prod_{\alpha n} d \sigma_{\alpha n}$ and the integral extends over all real fields. The positive definite "weight function" is

$W(\sigma)=\exp \left(-\frac{1}{2} \Delta \beta \sum_{\alpha n}\left|V_{\alpha}\right| \sigma_{\alpha n}^{2}\right)\left|\left\langle\Psi\left|U\left(N_{t}, 0\right)\right| \Psi\right\rangle\right|$.

Here, the one-body evolution operators are $U(k, j) \equiv$ $\prod_{n=j+1}^{k} U_{n}$, with $U_{n}=\exp \left(-\Delta \beta h_{n}\right)$, the one-body 
Hamiltonian being

$$
h_{n}=\sum_{\alpha} \epsilon_{\alpha} \mathcal{O}_{\alpha}+\sum_{\alpha} V_{\alpha} s_{\alpha} \sigma_{\alpha n} \mathcal{O}_{\alpha}
$$

where $s_{\alpha}=+1$ if $V_{\alpha}<0$ and $s_{\alpha}=i$ if $V_{\alpha}>0$. The remaining functions in the integrand are the "observable"

$$
O(\sigma) \equiv \frac{\left\langle\Psi\left|U\left(N_{t}, N_{t} / 2\right) O U\left(N_{t} / 2,0\right)\right| \Psi\right\rangle}{\left\langle\Psi\left|U\left(N_{t}, 0\right)\right| \Psi\right\rangle}
$$

and the "sign,"

$$
\Phi(\sigma) \equiv \frac{\left\langle\Psi\left|U\left(N_{t}, 0\right)\right| \Psi\right\rangle}{\left|\left\langle\Psi\left|U\left(N_{t}, 0\right)\right| \Psi\right\rangle\right|} .
$$

Similar expressions, valid for $N_{t}$ either even or odd, can be written for the grand canonical trace (with the introduction of chemical potentials) or for the canonical trace (using coherent states or an expansion in the fugacity) $[5]$.

The advantages of these expressions are manifest. The $h_{n}$ are one-body operators, so that the action of $U_{n}$ on a determinant is particularly simple. Indeed, the $\mathcal{O}_{\alpha}, h_{n}$, and $U_{n}$ can be represented as $N_{S} \times N_{S}$ matrices, while the determinant $|\Psi\rangle$ can be defined by $N_{V}$ single-particle wave functions (each a vector of dimension $N_{S}$ ). Hence all of the quantum mechanics can be done explicitly by matrix manipulation, while the integrals over the $\sigma_{\alpha n}$ can be done by standard Monte Carlo methods (e.g., the Metropolis algorithm). Of course, $N_{t}$ must be chosen large enough so that the $\Delta \beta \rightarrow 0$ limit is practically satisfied.

As noted above, our methods have several antecedents. Relative to simulations of the Hubbard model [3], the number of single-particle orbitals is considerably smaller than the typical number of lattice sites, and even relatively small fillings are of interest. Further, the shellmodel operators $\mathcal{O}_{\alpha}$ are fully nonlocal in the basis, rather than being at most nearest-neighbor hopping. Finally, proportionately smaller values of $\beta$ are of interest in the nuclear problem. As shown below, $\beta\left|V_{\alpha}\right| \lesssim 20$ can yield useful results, while for an on-site Hubbard repulsion of $1 \mathrm{eV}$, a temperature of $100 \mathrm{~K}$ implies $\beta\left|V_{\alpha}\right| \sim 120$. Other authors have presented path-integral calculations of the nuclear shell model [6]. However, these have generally been based on the uncontrolled static path approximation (SPA) in which the fields have no time dependence (harmonic corrections to the SPA have also been considered). Further, only the simplest schematic interactions have been treated.

To demonstrate our methods, we calculate groundstate properties of ${ }^{24} \mathrm{Mg}$ and ${ }^{48} \mathrm{Cr}$ via Eqs. (3)-(6), respectively, in the $s d$ - and $p f$-shell bases. While these calculations are not quite as demanding as those at the middle of the respective shells $\left({ }^{28} \mathrm{Si}\right.$ and $\left.{ }^{60} \mathrm{Zn}\right)$, these nuclei are deformed and hence present nontrivial problems. As noted above, Hamiltonians in the $s d$ shell can be diagonalized exactly, allowing a rigorous test of our methods; diagonalization of the ${ }^{48} \mathrm{Cr}$ Hamiltonian in the $p f$ space is at today's computational limit.

For these first calculations, we have used an interaction of the "pairing + multipole" form:

$$
V=V_{\text {pair }}+V_{\text {multi }} \text {. }
$$

This interaction is complicated enough to demonstrate the power of our methods, but is not meant to produce observables that can be compared directly to experiment. The pairing is taken to be of constant strength and isovector monopole character:

$$
V_{\text {pair }}=-G \sum_{j j^{\prime}}\left[a_{j}^{\dagger} \times a_{j}^{\dagger}\right]_{J=0, T=1} \cdot\left[\tilde{a}_{j^{\prime}} \times \tilde{a}_{j^{\prime}}\right]_{J=0, T=1} .
$$

For the multipole force, we take

$V_{\text {multi }}=\sum_{L=0,2,4} C_{L} f_{L}\left(r_{1}\right) f_{L}\left(r_{2}\right) Y_{L}\left(\hat{r}_{1}\right) \cdot Y_{L}\left(\hat{r}_{2}\right)$

where the radial functions are $f_{0}=1$ and

$$
f_{L>0}(r)=r^{L-1} \frac{d g}{d r}
$$

with $g$ a Woods-Saxon function. Two-body matrix elements of $V_{\text {multi }}$ were calculated in a harmonic oscillator single-particle basis. The values of the interaction parameters and single-particle energies were chosen to roughly reproduce the $T=1$ two-body matrix elements of the $s d$-shell Wildenthal interaction [2].

We note that our interaction is not as simple as it might appear. As we rewrite the pairing interaction in the form of Eq. (1) with the $\mathcal{O}_{\alpha}$ chosen as density operators, we must introduce all possible fields. This contrasts sharply with previous treatments of the nuclear shell model [6], where the forces were chosen so that only a small number of fields (five in the case of SPA for a quadrupole-quadrupole interaction) were needed. In our largest calculations, 9600 fields were required.

Of course, an interaction of the form (7)-(9) is not the most general possible. We have found that when a general residual interaction (e.g., the antisymmetrized twobody matrix elements of the Wildenthal interaction [2]) is arbitrarily linearized via the Hubbard-Stratonovich representation, the so-called "sign problem" can arise; that is, $\Phi(\sigma)$ in Eq. (6b) can be both positive and negative, so that the resulting cancellation leads to unacceptable statistical fluctuations. However, it is possible to prove that an interaction of the form (7)-(9), when applied to $T_{Z}=0$ nuclei, is completely free of the sign problem.

We have been able to show empirically that the flexibility in formulating the integral to be calculated (using varying proportions of the density or pairing breakups, and adding "nonphysical" symmetrized two-body matrix elements) can be used to mitigate the sign problem. While we can use this flexibility only in an ad hoc manner at present and do not fully understand the sources of (and solutions to) the sign problem for a general interaction, we are optimistic that a wide range of interactions will be tractable.

Figures 1 (a) and $1(\mathrm{~b})$ show, for ${ }^{24} \mathrm{Mg}$ and ${ }^{48} \mathrm{Cr}$, re- 

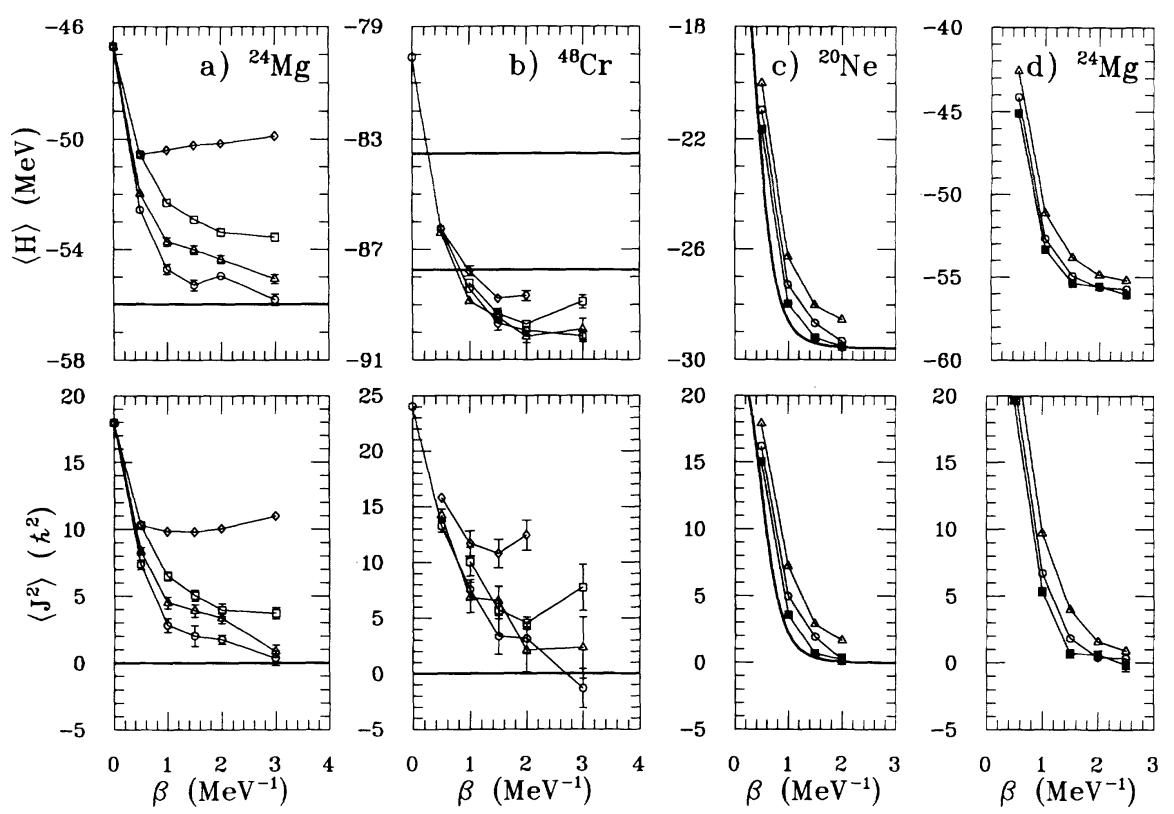

FIG. 1. $\langle H\rangle$ and $\left\langle J^{2}\right\rangle$ for selected $s d$ and $p f$ cases as functions of $\beta$. $\diamond$, SPA; $\square, \Delta \beta=0.5 ; \Delta, \Delta \beta=0.25 ; \circ, \Delta \beta=0.125$; 口, $\Delta \beta=0.0625 \mathrm{MeV}^{-1}$. (a) ${ }^{24} \mathrm{Mg}$ ground state; (b) ${ }^{48} \mathrm{Cr}$ ground state; (c) ${ }^{20} \mathrm{Ne}$, canonical ensemble; (d) ${ }^{24} \mathrm{Mg}$, canonical ensemble. Solid lines indicate the results of direct diagonalization, except for (b), where $\langle H\rangle$ for two different truncations of the model space are shown. Where not shown, error bars are smaller than the size of the plotting symbols. Each point corresponds to between 2000 and 5000 samples.

spectively, $\langle H\rangle$ and $\left\langle J^{2}\right\rangle$ as functions of $\beta$ for various $\Delta \beta$. The ${ }^{24} \mathrm{Mg}$ trial state was taken to be the prolate Hartree solution, while for ${ }^{48} \mathrm{Cr}$ we chose the maximally prolate trial state in only the $1 f_{7 / 2}$ orbital. There is a clear relaxation with increasing $\beta$ and a convergence as $N_{t}$ is increased (or $\Delta \beta$ decreased) at fixed $\beta$.

At large $\beta,\langle H\rangle$ for ${ }^{24} \mathrm{Mg}$ is in excellent agreement with the results of direct diagonalization, given by the solid lines. For ${ }^{48} \mathrm{Cr}$ in Fig. 1(b), we cannot diagonalize the Hamiltonian in the full model space; however, one can compare with $\langle H\rangle$ calculated at two levels of truncation of the model space $\left(f_{7 / 2}^{8}\right.$ and $f_{7 / 2}^{8}$ plus two-particle-twohole excitations) and note that our result is below these two. We therefore believe that we have successfully calculated the ground state of ${ }^{48} \mathrm{Cr}$. To our knowledge, no calculation has ever before treated this nucleus in the full $p f$ shell. We can also, in principle, calculate any $n$-body observable of the ground state. Note that for both nuclei, $\left\langle J^{2}\right\rangle$ is large for small $\beta$ (because of the deformed trial state), but vanishes for large $\beta$ (because of the $J=0$ ground state), and that the SPA is quite inadequate.

Using the methods described in Ref. [5], we can also calculate observables in the canonical (fixed-number, fixed-temperature) ensemble. Such calculations, which do not require a trial state, are essential for describing a nucleus at finite temperature in view of the large number fluctuations in the grand canonical ensemble. Results for ${ }^{20} \mathrm{Ne}$ are shown in Fig. 1(c), where they are also compared with direct diagonalization. Results for ${ }^{24} \mathrm{Mg}$, which cannot be obtained by other methods, are shown in Fig. 1(d).

An important point is the relatively gentle scaling of our methods with $N_{V}$ and/or $N_{S}$, as illustrated in Table I. The time to construct the Hamiltonian matrix and find the lowest-lying eigenvalues using the Lanczos algorithm increases rapidly with the dimension of the manybody basis, and memory requirements also grow dramatically with the basis size. Typical shell-model codes, such as OXBASH [7] or the Glasgow-Los Alamos-Seattle code $[8]$, have been used successfully throughout the entire $s d$ shell. However, because of computational time and memory demands, calculations in the $p f$ shell require either further truncations of the model space or restriction to relatively few valence nucleons. For example, a full $p f$ shell calculation of ${ }^{48} \mathrm{Cr}$, with only eight valence nucleons, would require on the order of 2 Gbyte mass storage. ${ }^{60} \mathrm{Zn}$ would require in excess of 1000 times more memory and, from that consideration alone, is presently impossible to treat exactly by diagonalization in the full $p f$ shell.

In contrast, Monte Carlo calculations scale with the number of auxiliary fields to be integrated over, which is, in turn, the square of the number of single-particle orbits (the number of possible density operators), 144 and 400 for the $s d$ and $p f$ shells, respectively. (The sixfold increase in time from the $s d$ to the $p f$ shell is due not only to this factor, but also to the longer correlation times in the Metropolis Monte Carlo sampling.) Fur-. thermore, because the Monte Carlo integration involves statistically independent samples, it is well suited to par- 
TABLE I. Computational time for shell-model calculations.

\begin{tabular}{cccc}
\hline \hline Nucleus & Basis size $^{\mathrm{b}}$ & \multicolumn{2}{c}{ Time (i860 node hours) } \\
$\left(N_{S} / N_{V}\right)^{\mathrm{a}}$ & $J=0, T=0$ & Diagonalization $^{\mathrm{c}}$ & Monte Carlo $^{\mathrm{d}}$ \\
\hline${ }^{24} \mathrm{Mg}$ & 325 & $0.04^{\mathrm{e}}$ & 607 \\
$(24 / 8)$ & $(28,503)$ & & \\
${ }^{28} \mathrm{Si}$ & 839 & $0.4^{\mathrm{e}}$ & 670 \\
$(24 / 12)$ & $(93,710)$ & & \\
${ }^{48} \mathrm{Cr}$ & 9741 & $\sim 40^{\mathrm{f}}$ & 3353 \\
$(40 / 8)$ & $(1963461)$ & & \\
${ }^{60} \mathrm{Zn}$ & 5053574 & $\sim 4 \times 10^{6 \mathrm{f}}$ & 3558 \\
$(40 / 20)$ & $\left(2.5 \times 10^{9}\right)$ & & \\
\hline \hline
\end{tabular}

${ }^{a} N_{S}$ is the number of single-particle orbits and $N_{V}$ is the number of valence nucleons.

${ }^{\mathrm{b}}$ In parentheses are the total number of $J_{Z}=0 \mathrm{~m}$-scheme Slater determinants from which $J=0, T=0$ states are projected.

${ }^{\mathrm{c}}$ Time to compute only the ground state.

${ }^{\mathrm{d}}$ Time for 3000 samples, giving the precision showin in Fig. 1, with $N_{t}=24$ and with adequate decorrelation of the Metropolis random walk. No extraordinary effort has been made to optimize performance.

${ }^{\mathrm{e}}$ Scaled from OXBASH calculations on VAX 3100 workstation. ${ }^{\mathrm{f}}$ Estimate using the Glasgow-Los Alamos-Seattle code [8].

allel computer architectures. The Monte Carlo calculations presented here were performed on the Intel Touchstone Gamma and Delta parallel supercomputers, with 64 and 512 nodes, respectively. Each of these nodes is an i860 processor that runs our code at a speed of 5 double-precision Mflops. The number of node hours for each calculation is given in the third column of Table I. ${ }^{60} \mathrm{Zn}$ calculations are clearly feasible (indeed, we have done them) and even a full $s d-p f$ calculation would be possible.

Future work will include not only a further investigation of the sign problem and use of more general residual interactions, but also applications to partition functions and level densities, strength functions (e.g., the $E 1$, $E 2$, and Gamow-Teller responses), high-spin nuclei, and problems in nuclear astrophysics.

We are grateful for discussions with other members of the Caltech nuclear theory group. C. W. Johnson and W. E. Ormand acknowledge Caltech Divisional and DuBridge postdoctoral fellowships, respectively. This work was supported in part by the National Science Foundation (Grants No. PHY90-13248 and No. PHY9115574).

[1] B. A. Brown and B. H. Wildenthal, Annu. Rev. Nucl. Part. Sci. 38, 29 (1988); R. D. Lawson, Theory of the Nuclear Shell Model (Clarendon, Oxford, 1980).

[2] B. H. Wildenthal, in Progress in Particle and Nuclear Physics, edited by D. H. Wilkinson (Pergamon, Oxford, 1984), Vol. 11, p. 5.

[3] S. R. White, D. J. Scalapino, R. L. Sugar, E. Y. Loh, J. E. Gubernaitis, and R. T. Scalettar, Phys. Rev. B 40, 506 (1989).

[4] G. Sugiyama and S. E. Koonin, Ann. Phys. (N.Y.) 168, 1 (1986).

[5] G. H. Lang, C. W. Johnson, W. E. Ormand, and S. E. Koonin (to be published).

[6] P. Arve, G. Bertsch, B. Lauritzen, and G. Puddu, Ann. Phys. (N.Y.) 183, 309 (1988); B. Lauritzen, P. Arve, and G. F. Bertsch, Phys. Rev. Lett. 61, 2835 (1988); B. Lauritzen and G. F. Bertsch, Phys. Rev. C 39, 2412 (1989); B. Lauritzen, G. Puddu, P. F. Bortignon, and R. A. Broglia, Phys. Lett. B 246, 329 (1990); G. Puddu, P. F. Bortignon, and R. A. Broglia, Phys. Rev. C 42, R1830 (1990); B. Lauritzen and J. W. Negele, Phys. Rev. C 44, 729 (1991); G. Puddu, P. F. Bortignon, and R. A. Broglia, Ann. Phys. (N.Y.) 206, 409 (1991); Y. Alhassid and B. W. Bush, Los Alamos Report No. LA-UR92-787 (to be published).

[7] W. D. M. Rae, A. Etchegoyen, and B. A. Brown, OXBASH, The Oxford-Buenos Aires-MSU shell-model code, Michigan State University Cyclotron Laboratory Report No. 524. OxBASH takes a set of $J_{Z}=J$ and $T_{Z}=T$ $m$-scheme Slater determinants and projects out a complete set of basis states that diagonalize $J$ and $T$; the Hamiltonian, which is block diagonal in this basis, is then more easily diagonalized.

[8] W. Haxton (private communication). The Glasgow-Los Alamos-Seattle code has not been described in detail in the literature, but its results can be found in, for example, W. C. Haxton and C. W. Johnson, Phys. Rev. Lett. 65, 1325 (1990), or J. Engel, W. C. Haxton, and P. Vogel, Institute for Nuclear Theory Report No. 40561-078-INT9207-12 (to be published). Note that, in contrast to OXBASH, this code does not initially project eigenstates of $J$ and $T$. 